\section{Combined treatment with ketoconazole and luteinising hormone releasing hormone analogue: a novel approach to resistant progressive prostatic cancer}

Adenocarcinoma of the prostate is androgen dependent in $80 \%$ of patients. Most circulating testosterone is secreted by the testes, but the adrenal glands contribute appreciably towards production of androgens. Conventional endocrine management is currently orientated towards suppressing testicular production of testosterone either directly by orchidectomy or by treatment with oestrogen.

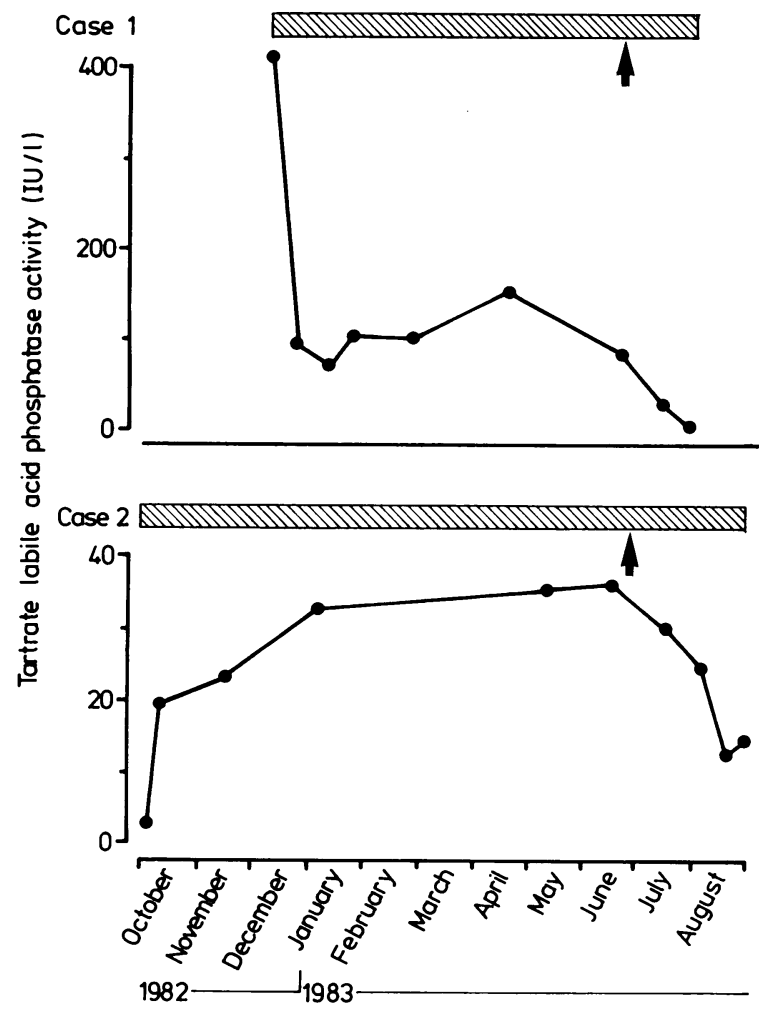

Tartrate labile acid phosphatase activity in two patients treated with ketoconazole after they had relapsed while taking an analogue of luteinising hormone releasing hormone. Hatched areas indicate treatment with the analogue; arrows show start of treatment with ketoconazole. The second patient had been treated for three months before October 1982, during which time tartrate labile acid phosphatase activity had remained below $10 \mathrm{IU} / 1$.

Recently superactive analogues of the hypothalamic peptide gonadotrophin releasing hormone have been used to inhibit pituitary secretion of luteinising hormone and thus to suppress gonadal production of testosterone. ${ }^{1}$ As adrenal production of androgens is independent of this axis, patients who relapse while receiving conventional treatment may not have escaped total endocrine control. It follows therefore that suppression of adrenal androgen production might be beneficial.

Ketoconazole is an oral imidazole drug in routine use as a systemic antifungal agent. It has recently been found to block both gonadal and adrenal synthesis of androgens. ${ }^{2}$ We report on two patients with advanced metastatic disease who relapsed despite achieving serum concentrations of testosterone seen after castration but showed early improvement when given ketoconazole.

\section{Case reports}

Case 1-Metastatic, poorly differentiated adenocarcinoma of the prostate was diagnosed in a 77 year old man. At presentation his tartrate labile acid phosphatase activity was $424 \mathrm{IU} / 1$ (normal $<2.5 \mathrm{IU} / \mathrm{l})$. He showed an initial subjective and objective response to treatment with a luteinising hormone releasing hormone analogue (ICI 118630$),{ }^{1}$ but after six months his acid phosphatase activity was still raised at $76 \cdot 3 \mathrm{IU} / \mathrm{l}$. Endocrine assessment ${ }^{1}$ at this stage showed continued effective suppression of the pituitary gonadal axis (testosterone concentration $1.16 \mathrm{nmol} / 1(0.33 \mathrm{ng} / \mathrm{ml}))$. Treatment with ketoconazole $200 \mathrm{mg}$ four times daily was added and resulted in a considerable fall in tartrate labile acid phosphatase activity (figure).

Case 2-Adenocarcinoma of the prostate had been diagnosed six years previously in a 72 year old man. Temporary remission had been achieved by bilateral orchidectomy, but in July 1982 a bone scan showed his condition to be deteriorating and his acid phosphatase activity was rising. A temporary symptomatic and clinical response was observed during treatment with an analogue of luteinising hormone releasing hormone, ${ }^{1}$ but in June 1983 progressive disease was again established despite maintained suppression of testosterone concentrations $(<1.0 \mathrm{nmol} / 1(0.29 \mathrm{ng} / \mathrm{ml}))$. After the addition of ketoconazole to treatment $(200 \mathrm{mg}$ four times daily rising to $400 \mathrm{mg}$ four times daily) serum tartrate labile acid phosphatase activity fell (figure).

\section{Comment}

Ketoconazole is used routinely as a systemic antifungal agent. Side effects are rare but include mild gastrointestinal disturbances, rashes, and hepatotoxicity. ${ }^{3}$ The drug's ability to interfere with both gonadal and adrenal synthesis of androgens has only recently been identified. ${ }^{2}$ Single dose studies have shown effective suppression of testosterone within eight hours and a compensatory rise in serum luteinising hormone concentration.

Relapse after remission induced by endocrine manipulation is a feature of prostatic carcinoma and has been considered to be a result of tumour cells losing their androgen dependency. Adrenal production of androgens, however, has largely been ignored, because the drugs available to suppress it are not well tolerated in high doses. Ketoconazole might, therefore, provide a useful further approach in managing these patients, and indeed a preliminary case has been reported in which ketoconaz le alone was used to treat a prostatic tumour. $^{5}$ Peripheral suppression of androgen synthesis, however, results in a compensatory rise in gonadotrophins, which may necessitate increasing doses of the drug to maintain suppression against increased pituitary drive. In addition, gonadotrophins are reported to be trophic factors to prostatic tumours. Thus an analogue of luteinising hormone releasing hormone and ketoconazole might be an ideal combination for total inhibition of androgen production. The response of our two patients, who had relapsed despite achieving testosterone concentrations seen after castration, indicates that this novel combined approach to advanced progressive prostatic tumours may be effective.

JMA is in receipt of a Wellcome Trust training fellowship.

${ }^{1}$ Allen JM, O'Shea JP, Mashiter K, Williams G, Bloom SR. Advanced carcinoma of the prostate: treatment with a gonadotrophin releasing hormone agonist. Br Med $\mathcal{f} 1983 ; 286: 1607-9$.

${ }^{2}$ Pont A, Williams PL, Azhar S, et al. Ketoconazole blocks testosterone synthesis. Arch Intern Med 1982;142:2137-40.

${ }^{3}$ Restrepo A, Stevens DA, Utz JP, eds. First international symposium on ketoconazole. Rev Infect Dis 1980;2:519-699.

${ }^{4}$ Pont A, Williams PL, Azhar S, et al. Ketoconazole blocks adrenal steroid synthesis. Arch Intern Med 1982;97:370-2.

5 Trachtenberg J, Halpern N, Pont A. Ketoconazole: a novel and rapid treatment for advanced prostatic cancer. $\mathcal{F}$ Urol 1983;130:152-3.

(Accepted 21 October 1983)

Departments of Medicine and Urology, Royal Postgraduate Medical School, Hammersmith Hospital, London W12

JANET M ALLEN, BSC, MRCP, research fellow and honorary senior registrar

DAVID J KERLE, FRCS, registrar

HOWARD WARE, MB, BS, senior house officer

ANDREW DOBLE, MB, BS, senior house officer

GORDON WILLIAMS, FRCS, consultant urologist

STEPHEN R BLOOM, DSC, FRCP, professor of endocrinology

Correspondence to: Professor S R Bloom. 